\title{
REMARKS ON THE CLASSICAL BANACH OPERATOR IDEALS
}

\author{
J. DIESTEL AND B. FAIRES
}

ABSTRACT. Sufficient conditions are given that the $\lambda$-tensor product of two operators be weakly compact.

Suppose $W, X, Y, Z$ denote Banach spaces and $W \otimes_{\lambda} X$ denotes the topological tensor product of $W$ and $X$ under the least reasonable tensor crossnorm $\lambda$. If $T: W \rightarrow Y$ and $S: X \rightarrow Z$ are continuous linear operators then a continuous linear operator $W \otimes_{\lambda} X \rightarrow Y \otimes_{\lambda} Z$ is induced which may or may not share certain special properties enjoyed by $T$ and $S$. The present note is concerned with the classical Banach operator ideal of weakly compact operators. While the $\lambda$-tensor product of weakly compact linear operators need not be weakly compact, if either of the operators in question is compact (Theorem 2) or if one of the operator's domains is a $C(K)$ - or an $L_{1}(\mu)$-space then the $\lambda$-tensor product of weakly compact operators is again a weakly compact operator (Theorem 4).

In proving that the $\lambda$-tensor product of a weakly compact operator and a compact operator is again weakly compact no real use is made of weak compactness; indeed, Theorem 2 shows that for any classical injective Banach operator ideal an analogous statement holds.

A basic tool used in the proof of Theorem 4 is a recent, as yet unpublished result of W. J. Davis, T. Figiel, W. B. Johnson, and A. Pełczyński which states that every weakly compact linear operator between Banach spaces factors through a reflexive Banach space. We wish to thank Professors Davis, Figiel, Johnson, and Pełczyński for communicating their result. We also wish to thank Professor D. R. Lewis for conversations which led to the present proof of Theorem 4; this proof avoids the use of the representation theory of weakly compact operators on $C(K)$ - and $L_{1}(\mu)$-spaces which we originally employed in proving Corollary 5.

Let $I$ denote a classical Banach operator ideal; i.e., for each pair of Banach spaces $X, Y, I(X ; Y)$ is a closed subspace of $L(X ; Y)$ in the uniform norm topology containing the finite rank operators $F(X ; Y)$ from $X$ to $Y$ and possessing the ideal property that if $W, Z$ are Banach spaces and $T: W \rightarrow X$, $R: Y \rightarrow Z$ are bounded linear operators and $S \in I(X ; Y)$, then $R S T: W \rightarrow Z$ is a member of $I(W ; Z)$. The reader is referred to [12] for a rather complete discussion of Banach operator ideals-classical and otherwise.

Among the classical examples of such structures one finds the classes of

Received by the editors October 16, 1973 and, in revised form, January 31, 1974. AMS (MOS) subject classifications (1970). Primary 47A05. 
compact operators [1], weakly compact operators [1], strictly singular operators ([9], [10]), strictly cosingular operators ([7], [10]), completely continuous operators $(T \in L(X ; Y)$ is said to be completely continuous if $T$ maps weakly convergent sequences into norm convergent sequences), Dunford-Pettis operators (weakly compact completely continuous operators), weak Cauchy operators $(T \in L(X ; Y)$ is said to be weak Cauchy whenever given a bounded sequence $\left(x_{n}\right) \subseteq X,\left(T x_{n}\right)$ has a weak Cauchy subsequence), and the unconditionally converging operators [8].

We say that a classical Banach operator ideal $I$ is injective [12] whenever given any bounded linear operator $T: X \rightarrow Y$ and any $Z \supseteq Y$ (isomorphically) then $T \in I(X ; Y)$ if and only if $T \in I(X ; Z)$. Among the aforementioned examples for $I$, all but the strictly cosingular operator ideal are examples of injective classical Banach operator ideals.

That the strictly cosingular operator ideal is not injective is seen by considering any isomorphism $T$ of $c_{0}$ into $C[0,1]$. Clearly, such a $T$ is not strictly cosingular, since $T c_{0}$ is, by Sobczyck's theorem [7], complemented in $C[0,1]$. Hence, the natural choices of $\varphi, \psi$ allow one to complete the diagram

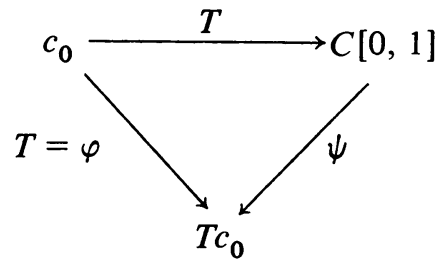

with $\varphi, \psi$ both linear, continuous, onto maps. We now embed $C[0,1]$ into $L_{\infty}[0,1]$. But every operator $S: c_{0} \rightarrow L_{\infty}[0,1]$ is strictly cosingular. In fact, if $E$ is any Banach space for which there exist epimorphisms $\varphi: c_{0} \rightarrow E, \psi$ : $L_{\infty}[0,1] \rightarrow E$, then as a quotient of $c_{0}, E$ is separable. However, by one of Grothendieck's theorems ([2, pp. 169-170]) separable quotients of $L_{\infty}[0,1]$ are reflexive. Thus $E$ is a reflexive quotient of $c_{0}$, i.e., $\varphi$ is weakly compact; but weakly compact linear operators on $c_{0}$ are compact. Thus $E$ is a compact quotient of $c_{0}$, i.e., $\operatorname{dim} E<\infty$.

REMARK. Using the results of [11] and [13] one can show that if $\Omega_{1}$ is a dispersed compact Hausdorff space and $\Omega_{2}$ is an $F$-space then every simultaneous Banach space quotient of $C\left(\Omega_{1}\right)$ and $C\left(\Omega_{2}\right)$ is finite dimensional. Hence every $T: C\left(\Omega_{1}\right) \rightarrow C\left(\Omega_{2}\right)$ or $T: C\left(\Omega_{2}\right) \rightarrow C\left(\Omega_{1}\right)$ is strictly cosingular. Whether or not this is so for any $\Omega_{2}$ such that $C\left(\Omega_{2}\right)$ is a Grothendieck space is not known.

THeOREM 1. Let I be a classical Banach operator ideal. Denote, for any pair of Banach spaces $X, Y$, by $J_{I}^{\lambda}(X ; Y)$ the class of all bounded, linear operators $T: X \rightarrow Y$ such that if $X_{1}, Y_{1}$ are Banach spaces and $S \in I\left(X_{1} ; Y_{1}\right)$ then $T \otimes_{\lambda} S \in I\left(X \otimes_{\lambda} X_{1} ; Y \otimes_{\lambda} Y_{1}\right)$ (here $\lambda$ denotes the least reasonable norm of [4]). Then $J_{I}^{\lambda}$ is a classical Banach operator ideal contained in $I$.

Proof. Let $T, S \in J_{I}^{\lambda}(X ; Y)$ and suppose $R: X_{1} \rightarrow Y_{1}$ is in $I\left(X_{1} ; Y_{1}\right)$. Then $T \otimes_{\lambda} R, S \otimes_{\lambda} R$ are in $I\left(X \otimes_{\lambda} X_{1} ; Y \otimes_{\lambda} Y_{1}\right)$ so 


$$
(T+S) \otimes_{\lambda} R=\left(T \otimes_{\lambda} R\right)+\left(S \otimes_{\lambda} R\right)
$$

is also in $I\left(X \otimes_{\lambda} X_{1} ; Y \otimes_{\lambda} Y_{1}\right)$ which yields $T+S \in J_{I}^{\lambda}(X ; Y)$.

Now let $W, Z$ be Banach spaces and let $T \in L(W, X), S \in J_{I}^{\lambda}(X, Y)$ and $R \in L(Y, Z)$. Suppose $U: X_{1} \rightarrow Y_{1}$ is a member of $I\left(X_{1} ; Y_{1}\right)$. Then the diagram

$$
\begin{gathered}
W \otimes_{\lambda} X_{1} \stackrel{R S T \otimes_{\lambda} U}{\longrightarrow} Z \otimes_{\lambda} Y_{1} \\
T \otimes_{\lambda} \operatorname{id}_{X_{1}} \downarrow \\
X \otimes_{\lambda} X_{1} \stackrel{{ }^{2} \otimes_{\lambda} \mathrm{id}_{Y_{1}}}{S \otimes_{\lambda} U} Y \otimes_{\lambda} Y_{1}
\end{gathered}
$$

commutes showing that $R S T \otimes_{\lambda} U$ is in $I\left(W \otimes_{\lambda} X_{1} ; Z \otimes_{\lambda} Y_{1}\right)$ and hence $R S T \in J_{J}^{\lambda}(W ; Z)$.

Thus $J_{I}^{\lambda}$ is an ideal.

We next show that $J_{I}^{\lambda}$ contains all the finite dimensional continuous operators. Indeed, let $T: X \rightarrow Y$ be a one dimensional linear continuous operator between the Banach spaces $X, Y$. Then $T$ is of the form $T x=f(x) y$ for some $f \in X^{\prime}$ and $y \in Y$. Now, if $X_{1}, Y_{1}$ are Banach spaces and $S$ $\in I\left(X_{1} ; Y_{1}\right)$, the following diagram commutes:

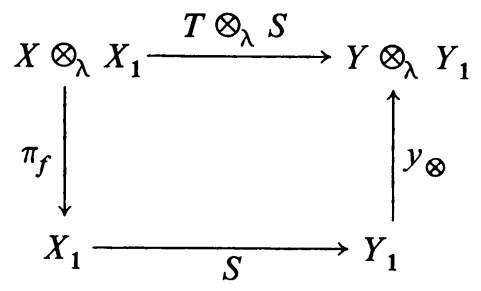

where $\pi_{f}\left(\sum_{i=1}^{n} x_{i} \otimes x_{i}^{(1)}\right)=\sum_{i=1}^{n} f\left(x_{i}\right) x_{i}^{(1)}$ and $y_{\otimes}\left(y^{1}\right)=y \otimes y^{1}$. Thus $T$ $\otimes_{\lambda} S \in I\left(X \otimes_{\lambda} X_{1} ; Y \otimes_{\lambda} Y_{1}\right)$ and hence $T \in J_{I}^{\lambda}(X ; Y)$. Linearity of $J_{I}^{\lambda}$ yields the containment of all the finite rank continuous operators in $J_{I}^{\lambda}$.

Finally, the inequalities

$$
\left\|T \otimes_{\lambda} S-T_{n} \otimes_{\lambda} S\right\|=\left\|\left(T-T_{n}\right) \otimes_{\lambda} S\right\| \leqslant\left\|T-T_{n}\right\| \cdot\|S\|
$$

show that $J_{I}^{\lambda}(X ; Y)$ is a closed subspace of $L(X ; Y)$ hence is a classical Banach operator ideal.

That $J_{I}^{\lambda} \subseteq I$ follows from the commutativity of the diagram

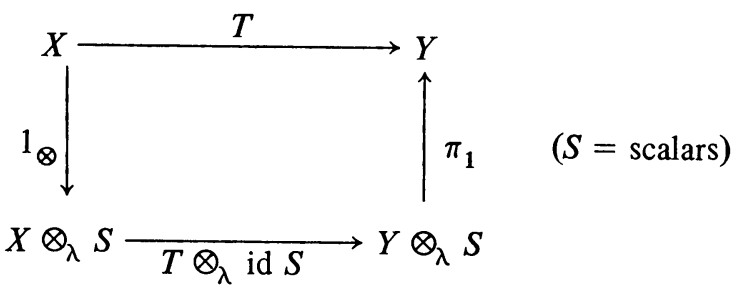

where $1_{\otimes}(x)=x \otimes 1$ and $\pi_{1}\left(\sum_{i=1}^{n} y_{i} \otimes r_{i}\right)=\sum_{i=1}^{n} r_{i} y_{i}$. 
Our next result contains as a very special case a theorem of J. R. Holub [6] in the case where $I$ is the compact operator ideal.

THEOREM 2. Suppose that I is an injective classical Banach operator ideal. Then $J_{I}^{\lambda}(X ; Y)$ is an injective classical Banach operator ideal; consequently, $J_{I}^{\lambda}$ contains the compact operator ideal.

Proof. The first assertion follows from the fact that $\lambda$ is an injective tensorial norm, i.e., if $Y \subseteq Z$ and $X$ is given then $X \otimes_{\lambda} Y \subseteq X \otimes_{\lambda} Z$ and $Y \otimes_{\lambda} X \subseteq Z \otimes_{\lambda} X$. This combined with the injectivity of $I$ yields the easy proof of the injectivity of $J_{I}^{\lambda}$.

The second assertion is a consequence of the following fact: every injective classical Banach operator ideal contains the compact operator ideal. Indeed, by the definition of operator ideal every ideal $I$ contains the class of finite rank operators. If $I$ is injective and $X, Y$ are Banach spaces then $Y$ is isometrically isomorphic to a subspace of some space $C(\Omega)$ of continuous real valued functions on a compact Hausdorff space $\Omega$. So, given $T: X \rightarrow Y$ compact, $T: X \rightarrow C(\Omega)$ is compact and hence the limit of finite rank operators (since by [3, pp. 180-186], $C(\Omega)$ possesses the metric approximation property). Therefore, $T \in I(X ; C(\Omega))$. By injectivity of $I, T \in I(X ; Y)$.

COROLlARY 3. The $\lambda$-tensor product of a compact operator with a

(i) (weakly) compact operator is (weakly) compact;

(ii) strictly singular operator is strictly singular;

(iii) unconditionally converging operator is unconditionally converging;

(iv) completely continuous operator is completely continuous;

(v) Dunford-Pettis operator is a Dunford-Pettis operator;

(vi) weak Cauchy operator is a weak Cauchy operator.

EXAMPLES. (i) The $\lambda$-tensor product of weakly compact operators need not be weakly compact; indeed, the identity operator id: $l_{2} \rightarrow l_{2}$ is weakly compact yet

$$
\text { id } \otimes_{\lambda} \mathrm{id}=\mathrm{id}_{l_{2} \otimes_{\lambda} l_{2}}: l_{2} \otimes_{\lambda} l_{2} \rightarrow l_{2} \otimes_{\lambda} l_{2}
$$

is not weakly compact by the well-known nonreflexivity of $l_{2} \otimes_{\lambda} l_{2}$.

(ii) The $\lambda$-tensor product of strictly singular operators need not be strictly singular; similarly, the $\lambda$-tensor product of unconditionally converging (strictly cosingular). The same example suffices in each case, namely, let $i$ denote the canonical inclusion of $l_{2}$ into $c_{0}$. Then $i \otimes_{\lambda} i: l_{2} \otimes_{\lambda} l_{2} \rightarrow c_{0} \otimes_{\lambda} c_{0}$ fixes the diagonal $e_{j} \otimes e_{j}\left(e_{j}\right.$ denotes the $j$ th unit vector) and hence is an isomorphism of the closed linear span of $\left\{e_{j} \otimes e_{j}: j=1,2, \ldots\right\}$ in $l_{2} \otimes_{\lambda} l_{2}$ with the closed linear span of $\left\{e_{j} \otimes e_{j}: j=1,2, \ldots\right\}$ in $c_{0} \otimes_{\lambda} c_{0}$. In each case, by Theorem 5.5 of [5], this space is a complemented subspace isomorphic to $c_{0}$. The assertions about $i \otimes_{\lambda} i$ now follow from the fact that $i: l_{2} \rightarrow c_{0}$ is a strictly singular, strictly cosingular, unconditionally converging operator; while clearly $i \otimes_{\lambda} i$ : $l_{2} \otimes_{\lambda} l_{2} \rightarrow c_{0} \otimes_{\lambda} c_{0}$ is none of these.

THEOREM 4. Let $W$ be a Banach space whose dual space possesses the approximation property and the Dunford-Pettis property. Let $T: W \rightarrow Y$ and 
$S: X \rightarrow Z$ be weakly compact linear operators. Then $T \otimes_{\lambda} S: W \otimes_{\lambda} X \rightarrow Y$ $\otimes_{\lambda} Z$ is a weakly compact linear operator.

Proof. We start by noting a consequence of the factorization result of Davis-Figiel-Johnson-Pełczyński; we may assume $X=Z$ is reflexive and $S$ is the identity on $X$. Indeed, if $R$ is a reflexive Banach space such that for some $A \in L(X ; R)$ and $B \in L(R ; Z)$ the diagram

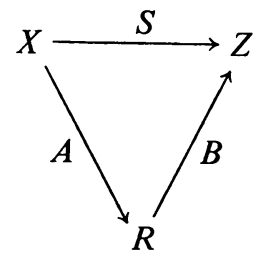

commutes, then the diagram

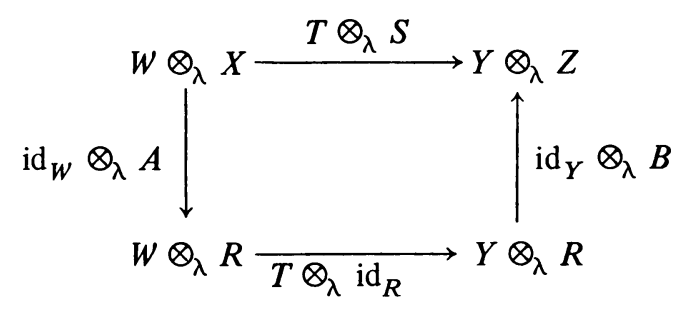

also commutes. Hence the weak compactness of $T \otimes_{\lambda} \mathrm{id}_{R}$ implies that of $T \otimes_{\lambda} S$.

So we are reduced to the question of tensoring a weakly compact operator on a space $W$ whose dual possesses the approximation and Dunford-Pettis properties with the (weakly compact) identity operator on a reflexive Banach space to yield (relative to the $\lambda$ cross-norm) a weakly compact operator.

Suppose we consider any Banach space $U$ whose dual possesses the approximation property and any reflexive Banach space $V$. Then the dual of $\left(U \otimes_{\lambda} V\right)$ is precisely the space of integral bilinear functionals $\left(B^{\gamma}(U, V)\right.$ in the notation of [4]) on $U \times V$. Identifying $B^{\gamma}(U, V)$ with a space of operators we have $\left(U \otimes_{\lambda} V\right)^{\prime}=$ integral operators from $U$ to $V^{\prime}$. By the Dunford-PettisPhillips theorem ([3, p. 134]), every integral operator into the reflexive space $V^{\prime}$ is nuclear; by the approximation assumption on $U^{\prime}$, the nuclear operators from $U$ to $V^{\prime}$ coincide with $U^{\prime} \otimes_{\gamma} V^{\prime}$. Thus under the above assumptions on $U$ and $V$ we have

$$
\left(U \otimes_{\lambda} V\right)^{\prime}=\left(U^{\prime} \otimes_{\gamma} V^{\prime}\right)
$$

Of course, by the universal mapping principle for $\gamma$ we have $\left(U^{\prime} \otimes_{\gamma} V^{\prime}\right)^{\prime}$ identifiable with $L\left(U^{\prime} ; V^{\prime \prime}\right)=L\left(U^{\prime} ; V\right)$.

Now let $T, S, W, X, Y, Z$ be as in the statement of the theorem. By the injectivity of the weakly compact operator ideal in order to show $T \otimes_{\lambda} S$ : $W \otimes_{\lambda} X \rightarrow Y \otimes_{\lambda} Z$ is weakly compact it suffices to show $T \otimes_{\lambda} S$ is weakly compact into any super space of $Y \otimes_{\lambda} Z$. In particular, embedding if necessary $Y$ into a $C(\Omega)$ space isometrically and using the injectivity of the $\lambda$ 
cross-norm we may and do assume that $Y$ and all of its duals possess the (metric) approximation property.

Thus in light of the above paragraphs, we are in the following situation: $W^{\prime}$ and $Y^{\prime}$ both possess the approximation property and $W^{\prime}$ also possesses the Dunford-Pettis property. $T: W \rightarrow Y$ is weakly compact and $R$ is a reflexive Banach space. We wish to show that

$$
T \otimes_{\lambda} \operatorname{id}_{R}: W \otimes_{\lambda} R \rightarrow Y \otimes_{\lambda} R
$$

is weakly compact, i.e., that $([1, \mathrm{VI}, 4.2])$

$$
\left(T \otimes_{\lambda} \operatorname{id}_{R}\right)^{\prime \prime}\left(L\left(W^{\prime} ; R\right)\right) \subseteq Y \otimes_{\lambda} R
$$

where by the third paragraph we have $L\left(W^{\prime} ; R\right)=\left(W \otimes_{\lambda} R\right)^{\prime \prime}$ and $L\left(Y^{\prime} ; R\right)$ $=\left(Y \otimes_{\lambda} R\right)^{\prime \prime}$.

The action of $\left(T \otimes_{\lambda} \mathrm{id}_{R}\right)^{\prime \prime}$ on $\varphi \in L\left(W^{\prime} ; R\right)$ is readily checked to be given by

$$
\left(T \otimes_{\lambda} \mathrm{id}_{R}\right)^{\prime \prime}(\varphi)=\varphi \cdot T^{\prime} \in L\left(Y^{\prime} ; R\right) .
$$

To show that $\varphi \cdot T^{\prime} \in Y \otimes_{\lambda} R$ we must show (by the approximation considerations for $Y$ ) that $\varphi \cdot T^{\prime}$ is a weak-star to weak, continuous compact linear operator from $Y^{\prime}$ to $R$. Weak star-weak continuity is immediate from $T^{\prime}$ 's weak star-weak continuity (VI, 4.7 of [1]) and the weak continuity of $\varphi(\mathrm{V}, 3.15$ of [1]). The compactness of $\varphi \cdot T^{\prime}$ follows from the fact that $T^{\prime}: Y^{\prime} \rightarrow W^{\prime}$ is weakly compact (VI, 4.18 of [1]) hence $T^{\prime}$ ( $Y^{\prime \prime}$ s unit ball) is relatively weakly compact in $W^{\prime}$ which, since $W^{\prime}$ possesses the Dunford-Pettis property and $\varphi: W^{\prime} \rightarrow R$ is necessarily weakly compact (and therefore maps relatively weakly compact subsets of $W^{\prime}$ into norm compact subsets of $R$ ), yields $\varphi \cdot T^{\prime}$ ( $Y^{\prime}$ unit ball) relatively compact in $R$.

COROLlary 5. If $W$ is either a $C(K)$-space ( $K$ a compact Hausdorff space) or an $L_{1}(\mu)$-space then $T \otimes_{\lambda} S: W \otimes_{\lambda} X \rightarrow Y \otimes_{\lambda} Z$ is weakly compact whenever $T$, $S$ are.

We thank the referee and Professor T. H. E. Skulker for providing the following lemma and example in answer to our questions in the original version of the paper.

Lemma. Suppose $T: X \rightarrow Y$ is onto and $T^{\prime} Y^{\prime}$ is complemented in $X^{\prime}$. Then $T \otimes_{\lambda} T: X \otimes_{\lambda} X \rightarrow Y \otimes_{\lambda} Y$ is onto.

Proof. We prove that $T \otimes_{\lambda} T$ is onto by showing that $\left(T \otimes_{\lambda} T\right)^{\prime}: L_{\gamma}\left(Y, Y^{\prime}\right)$ $\rightarrow L_{\gamma}\left(X, X^{\prime}\right)$ is an isomorphism, where $L_{\gamma}\left(X, X^{\prime}\right)$ denotes the integral operators from $X$ into $X^{\prime}$. Let $P: X^{\prime} \rightarrow T^{\prime} Y^{\prime}$ be a projection and recall that for $S \in L_{\gamma}\left(Y, Y^{\prime}\right),\left(T \otimes_{\lambda} T\right)^{\prime}(S)=T^{\prime} S T$. Since for $S \in L_{\gamma}\left(Y, Y^{\prime}\right)$, both $S T$ and $T^{\prime} S T$ are integral operators, we have the following diagram: 


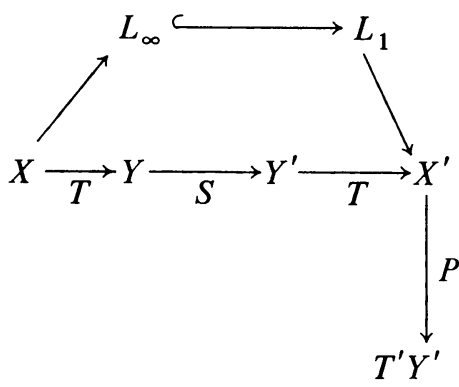

which shows that

$$
\|S T\|_{\gamma}=\left\|P T^{\prime} S T\right\|_{\gamma} \leqslant\|P\| \cdot\left\|T^{\prime} S T\right\|_{\gamma} .
$$

The dual diagram

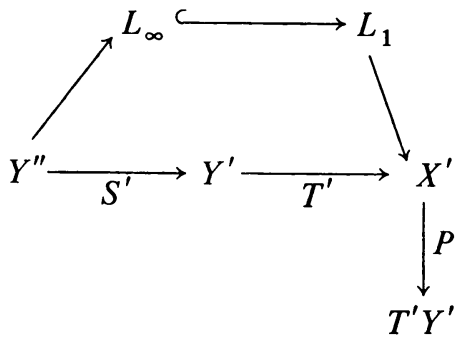

yields that

$$
\|S\|_{\gamma}=\left\|S^{\prime}\right\|_{\gamma}=\left\|P T^{\prime} S^{\prime}\right\|_{\gamma} \leqslant\|P\| \cdot\left\|T^{\prime} S^{\prime}\right\|_{\gamma}=\|S T\|_{\gamma} .
$$

Thus if $\left(T \otimes_{\lambda} T\right)^{\prime} S=T^{\prime} S T=0$ we have by (1) and (2) that $S=0$.

Using this result we give an example to show that the $\lambda$-tensor product of Dunford-Pettis type operators is not an operator of Dunford-Pettis type.

Let $X=\left(\sum_{n} l_{2}^{n}\right)_{l}$ and $T: X \rightarrow l_{2}$ be the map defined by $T e_{i}^{n}=e_{i}$, where $\left(e_{i}\right)_{i=1}^{n}$ is the unit vector basis for $l_{2}^{n}$ and $\left(e_{i}\right)$ is the unit vector basis for $l_{2}$. To show that $T$ is onto and $T^{\prime} l_{2}$ is norm one complemented in $X^{\prime}$, we show that $T$ admits local selections and use Stegall's local selection lemma ([14, Lemma 1]). If $G$ is any finite dimensional Banach space and $S: G \rightarrow l_{2}$, letting $f_{1}, f_{2}, \ldots, f_{n}(\operatorname{dim} G=n)$ denote an orthonormal basis of $S G,\left[\left(f_{j}\right)_{j=1}^{n}\right]$ is isometrically isomorphic to $l_{2}^{n}$. Letting $\hat{S}=S$ with $f_{i}=e_{i}, T$ admits a local selection with $\lambda=1$. It is clear that $X$ has the Dunford-Pettis property and $T$ is weakly compact. $T \otimes_{\lambda} T: X \otimes_{\lambda} X \rightarrow l_{2} \otimes_{\lambda} l_{2}$ is onto (by previous lemma) and hence not weakly compact by the nonreflexivity of $l_{2} \otimes_{\lambda} l_{2}$.

\section{REFERENCES}

1. N. Dunford and J. T. Schwartz, Linear operators. I: General theory, Pure and Appl. Math., vol. 7, Interscience, New York, 1958. MR 22 \#3302.

2. A. Grothendieck, Sur les applications linéaires faiblement compactes d'espaces du type $C(K)$, Canad. J. Math. 5 (1953), 129-173. MR 15, 438.

3. _ Produits tensoriels topologiques et espaces nucléaires, Mem. Amer. Math. Soc. No. 16 (1955). MR 17, 763. 
4. Resumé de le théorie mé trique des produits tensoriels topologiques, Bol. Soc. Mat. São Paulo 8 (1956), 1-79.

5. J. R. Holub, Tensor product bases and tensor diagonals, Trans. Amer. Math. Soc. 151 (1970), 563-579. MR 43 \# 5286.

6. Compactness in topological tensor products and operator spaces, Proc. Amer. Math. Soc. 36 (1972), 398-406. MR 48 \#4802.

7. A. Pełczyński, Projections in certain Banach spaces, Studia Math. 19 (1960), 209-228. MR 23 \# A3441.

8. Banach spaces on which every unconditionally converging operator is weakly compact, Bull. Acad. Polon. Sci. Sér. Sci. Math. Astronom. Phys. 10 (1962), 641-648. MR 26 \#6785.

9. - On strictly singular and strictly cosingular operators. I. Strictly singular and strictly cosingular operators in C(S)-spaces, Bull. Acad. Polon. Sci. Sér. Sci. Math. Astronom. Phys. 13 (1965), 31-36. MR 31 \# 1563.

10. - On strictly singular and strictly cosingular operators. II. Strictly singular and strictly cosingular operators in L(v)-spaces, Bull. Acad. Polon. Sci. Sér. Sci. Math. Astronom. Phys. 13 (1965), 37-41. MR 31 \#1564.

11. A. Pelczyński and Z. Semadeni, Spaces of continuous functions. III. Spaces $C(\Omega)$ for $\Omega$ without perfect subsets, Studia Math. 18 (1959), 211-222. MR 21 \#6528.

12. A. Pietsch, Theorie der Operatorenideale, Friedrich-Schiller-Universität, Jena, 1972.

13. H. P. Rosenthal, On complemented and quasi-complemented subspaces of quotients of $C(S)$ for Stonian S, Proc. Nat. Acad. Sci. U.S.A. 60 (1968), 1165-1169. MR 37 \#6740.

14. C. Stegall, Banach spaces whose duals contain $l_{1}(\Gamma)$ with applications to the study of dual $L_{1}(\mu)$ spaces (preprint).

Department of Mathematics, Kent State University, Kent, Ohio 44242

Department of Mathematics, Carnegie-Mellon University, Pittsburgh, Pennsylvania 15213 